\title{
Optimal Sensitivity Control of Piezoelectric Smart Truss Based on POS Algorithm
}

\author{
Xiangjie Chen \\ School of Mathematics, Southeast University, Nanjing 210000, China. \\ chenxj1218@foxmail.com
}

Keywords: Truss structure; modal sensor; particle swarm optimization algorithm.

\begin{abstract}
The optimal sizing design of truss structures is studied using the recently proposed particle swarm optimization algorithm (PSOA), in which the social behavior of birds is mimicked. Individual birds in a flock exchange information about their position, velocity and fitness, and the behavior of the flock is then influenced to increase the probability of migration to regions of high fitness. A simple approach is presented to accommodate the stress and displacement constraints during initial stages of the swarm searches. In this approach, increased social pressure, at the cost of cognitive learning, is exerted on infeasible birds to increase their rate of migration to feasible regions. Numerical results are presented for a number of well-known test functions, with dimensionality of up to 21 .
\end{abstract}

\section{Introduction}

With the development of space industry, the spacecraft is developing towards the direction of light weight and large scale. As a special structure, the truss structure has been used more and more widely. Because of its low stiffness and weak damping, the vibration problem is very prominent. For a long time, the truss is tied up. The problem of structural vibration control has always been the focus of research. Especially the emergence of piezoelectric ceramic actuators makes it possible to control active vibration of complex trusses. An adaptive structure is formed by combining the actuator and the sensor with the truss structure. It has a wide application prospect in the field of vibration control, noise suppression, shape control and precision positioning.

The optimal vibration control of piezoelectric smart truss is studied in this paper. Firstly, the dynamic equations of the piezoelectric intelligent truss structure are established by using the finite element theory. Then the optimal control theory is used to control the truss structure. The optimal control input is determined by minimizing the performance functional and solving the Rickety matrix algebraic equation. Finally, a simulation example of plane intelligent truss structure is given to verify the modeling process and the proposed vibration control algorithm.

\section{Dynamic Modeling of Piezoelectric Intelligent Truss Structure}

\subsection{Modeling of Piezoelectric Actuator.}

Piezoelectric ceramic stack is made up of laminated piezoelectric patches, and the piezoelectric equation of piezoelectric element is introduced in detail. The piezoelectric actuator produces axial expansion and displacement under the action of voltage. According to the actual structure of the piezoelectric actuator, neglecting the influence of the piezoelectric ceramic sheet connection and neglecting the influence of the sleeve's own stiffness, it can be simplified to a concentrated mass $\mathrm{M}$ and a spring series with a stiffness of $\mathrm{K}$. 


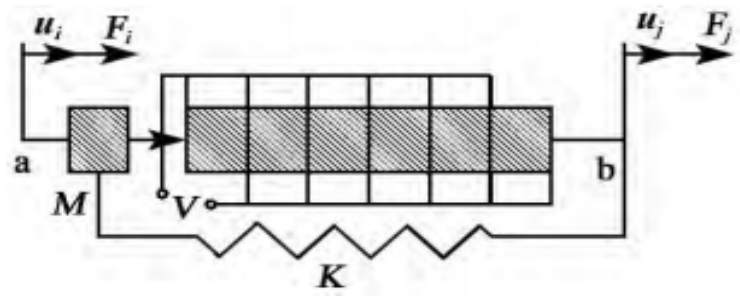

Fig 1. Mechanical model simplification of piezoelectric actuator.

The electromechanical coupling dynamic equation of axial expansion PZT piezoelectric element can be expressed as Hamilton variation principle:

$$
\int_{\mathrm{t}_{0}}^{t_{1}} \delta L d t+\int_{\mathrm{t}_{0}}^{t_{1}} \delta L d t=0
$$

The formula is: $\mathrm{L}=\mathrm{T} \mathrm{U}$ is Lagrange function, $\mathrm{T}$ is system kinetic energy, $\mathrm{U}$ is system potential energy; Delta $\mathrm{W}$ is virtual work for external load.

The dynamic equation of the piezoelectric actuator can be obtained from the upper formula:

$$
\left\{\begin{array}{l}
\boldsymbol{M}^{(a)} \ddot{\boldsymbol{u}}^{(a)}+\boldsymbol{K}^{(a)} \boldsymbol{u}^{(a)}+\boldsymbol{K}_{u v}^{(a)} \boldsymbol{\phi}=\boldsymbol{F}^{(a)} \\
\boldsymbol{K}_{v u}^{(a)} \boldsymbol{u}^{(a)}+\boldsymbol{K}_{v v}^{(a)} \boldsymbol{\phi}=-\boldsymbol{Q}^{(a)}
\end{array}\right.
$$

\subsection{Electromechanical Coupling Modeling of a Piezoelectric Smart Pole.}

The piezoelectric active rod is composed of two main parts and an intermediate piezoelectric actuator unit, as shown in Fig. 3, in which the main material unit equation is:

$$
\left[\begin{array}{cc}
2 m_{1} & m_{1} \\
m_{1} & 2 m_{1}
\end{array}\right]\left\{\begin{array}{l}
\ddot{u}_{l} \\
\ddot{u}_{i}
\end{array}\right\}+\left[\begin{array}{cc}
k_{1} & -k_{1} \\
-k_{1} & k_{1}
\end{array}\right]\left\{\begin{array}{l}
u_{1} \\
u_{1^{\prime}}
\end{array}\right\}=\left\{\begin{array}{c}
F_{1} \\
-F_{1^{\prime}}
\end{array}\right\}
$$

The mass matrix and stiffness matrix are assembled to obtain the global stiffness matrix:

$$
\left\{\begin{array}{l}
\boldsymbol{M}^{c} \ddot{\boldsymbol{u}}^{c}+\boldsymbol{K}^{c} \boldsymbol{u}^{c}+\boldsymbol{K}_{w v}^{c} \phi=\boldsymbol{F}^{c} \\
\boldsymbol{K}_{u v}^{c} \boldsymbol{u}^{c}-\boldsymbol{K}_{v v}^{c} \phi=-Q^{c}
\end{array}\right.
$$

In order to reduce the dimension of the system model, the degree of freedom can be reduced by using the Guyana static condensation method:

$$
\boldsymbol{u}^{e}=\boldsymbol{H} \boldsymbol{\delta}^{e}+\boldsymbol{M} \boldsymbol{\phi}
$$

The electromechanical coupling dynamic equation of the driving rod can be obtained by combining the upper form:

$$
\left\{\begin{array}{l}
\boldsymbol{M}^{e} \ddot{\boldsymbol{\delta}}^{e}+\boldsymbol{K}^{e} \boldsymbol{\delta}^{e}+\boldsymbol{K}_{u v}^{e} \boldsymbol{\phi}=\boldsymbol{F}^{e} \\
\boldsymbol{K}_{v v}^{e} \boldsymbol{\delta}^{e}-\boldsymbol{K}_{w v}^{e} \boldsymbol{\phi}=-Q^{e}
\end{array}\right.
$$

By means of polycondensation transformation, the equation of the piezoelectric active rod has the same form as that of the common rod element. Based on the above derivation, the piezoelectric active member model is obtained. By coordinate transformation, the mass matrix and stiffness matrix of each element are transformed into the characteristic matrix in the overall coordinate system. The dynamic equations of the piezoelectric truss structure are obtained:

$$
\widetilde{\boldsymbol{M}} \ddot{\boldsymbol{\delta}}+\widetilde{\boldsymbol{K}} \boldsymbol{\delta}+\widetilde{\boldsymbol{K}}_{u v} \phi=\widetilde{\boldsymbol{F}}
$$

$\widetilde{\boldsymbol{M}}=\sum \boldsymbol{T}^{e} \boldsymbol{M}^{e} \boldsymbol{T}^{e \mathrm{~T}}$ Is the total mass matrix of the system; $\widetilde{\boldsymbol{K}}=\sum \boldsymbol{T}{ }^{e} \boldsymbol{K}^{e} \boldsymbol{T}^{e \mathrm{~T}}$ is the overall stiffness matrix of the system; $\widetilde{\boldsymbol{K}}_{w v}=\sum \boldsymbol{T}^{e} \boldsymbol{K}^{u v}$ is the overall coupling stiffness matrix; $\widetilde{\boldsymbol{F}}=\sum \boldsymbol{T}^{e} \boldsymbol{F}^{e}$ is the total physical vector; $\boldsymbol{T}$ is the coordinate transformation matrix.

\section{Modal Sensor Design}

To design the modal sensor, employing Green formula, we have 


$$
\begin{aligned}
q(t) & =-\sum_{i=1}^{\infty} \sum_{j=1}^{\infty} \eta_{i j}\left[\iint_{s} W_{i j} \nabla^{2} F_{s} \mathrm{~d} x \mathrm{~d} y\right. \\
& \left.+\int_{\Gamma} W_{i j} \frac{\partial F_{s}}{\partial n} \mathrm{~d} \Gamma-\int_{\Gamma} F_{s} \frac{\partial W_{i j}}{\partial n} \mathrm{~d} \Gamma\right]
\end{aligned}
$$

If the spatial distribution function of the sensor layer satisfies:

$$
\begin{gathered}
F_{s}(x, y)=A_{s} D \sum_{k=1}^{K} \sum_{l=1}^{L} A_{k l}^{s} \nabla^{2} W_{k l}(x, y)+C_{s}, \quad x, y \in S \\
F_{s}=0, \quad \frac{\partial F_{s}}{\partial n}=0, \quad x, y \in \Gamma_{(*)}
\end{gathered}
$$

The following equations can be obtained:

$$
\begin{aligned}
& q(t)=-A_{s} \sum_{k=1}^{K} \sum_{l=1}^{L} A_{k l}^{s} \omega_{k l}^{2} \eta_{k l}(t) \\
& I(t)=-A_{s} \sum_{k=1}^{K} \sum_{l=1}^{L} A_{k l}^{s} \omega_{k l}^{2} \dot{\eta}_{k l}(t)
\end{aligned}
$$

Where $I(\mathrm{t})$ the current is output of the sensor, $A_{\mathrm{s}}$ and $C_{\mathrm{s}}$ are constants. Equations show that the sensor layer with the distribution function satisfying Eq. $\left(^{*}\right)$ is only sensitive to the selected modes, and, therefore, such a piezoelectric sensor layer becomes a modal sensor. After obtaining the spatial distribution of the sensor layer from Eq. $\left(^{*}\right)$ ), its thickness distribution function can be determined by following the similar procedure with the actuator. In fact, if the plate is clamped along its four sides the boundary condition in Eq. (*) can be ignored. For a simply supported plate the second boundary condition can be ignored too, and the first boundary condition can be satisfied by choosing $C_{S}=0$. In this case, to keep the thickness positive, the polarity direction of the parts with negative thickness should be reversed.

\section{Experiment and Result Analysis}

As an illustrative example, consider a $1 \mathrm{~m} \times 0.7 \mathrm{~m} \times 1 \mathrm{~mm}$ simply supported rectangular plate onto which one piezoceramic actuator layer and one piezoceramic sensor are bonded. The actuator and the sensor layers are made of the same lead zircon ate titan ate (PZT) material, and $\mathrm{e}_{31}^{a}=\mathrm{e}_{31}^{s}=23: 31 \mathrm{~N} / \mathrm{Vim}$. The Young's modulus of the host plate is $210 \mathrm{GA}$, and its mass density is $8000 \mathrm{~kg} / \mathrm{m} 3$. The first five $(11 ; 21 ; 12 ; 31 ; 22)$ modes are selected to be controlled by using the modal sensor and the modal actuator. The maximum thickness of the modal actuator is less than $0.12 \mathrm{~mm}$. The modal sensor is designed with the parameters $A_{11}^{S}=1.0, A_{21}^{S}=0.7, A_{12}^{S}=0.6, A_{31}^{S}=0.5, A_{22}^{S}=0.4$ so that its maximum thickness is less than $0.1 \mathrm{~mm}$.
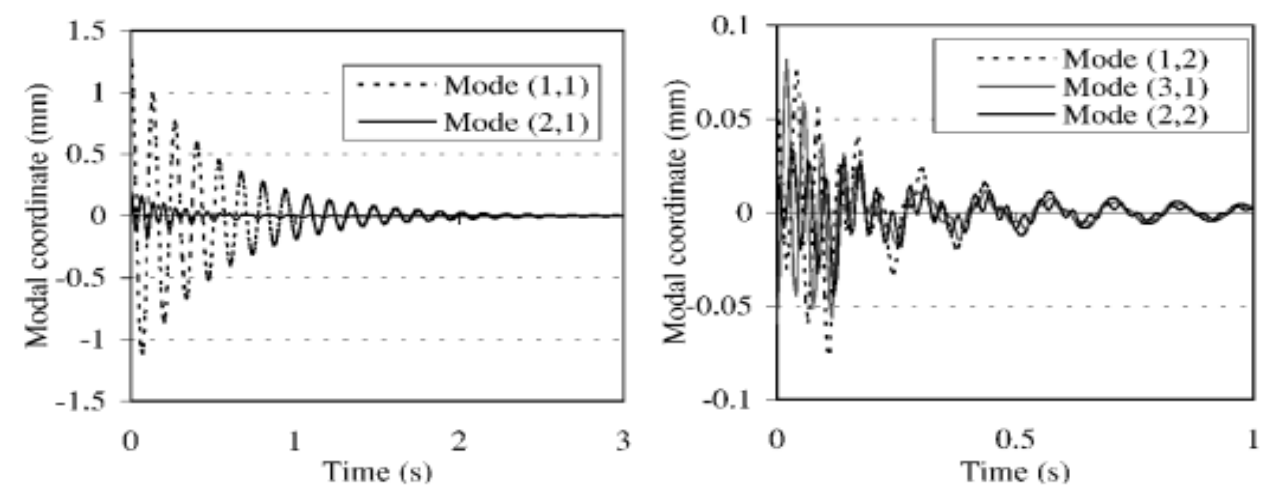

Fig 2. Five controlled modes of the smart plate. 


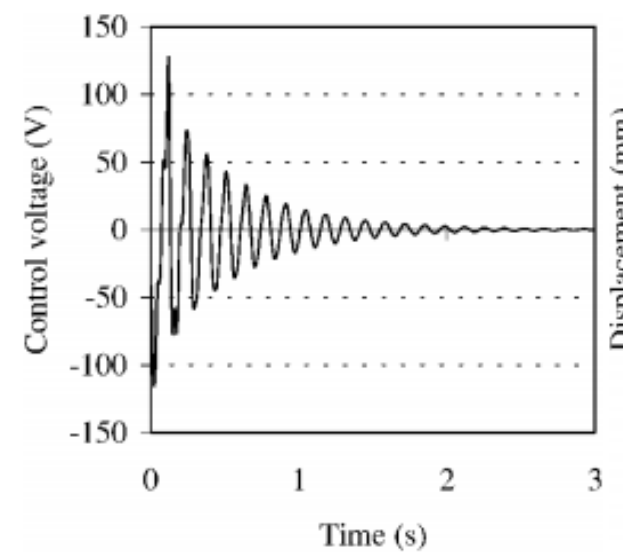

(a)Central displacement

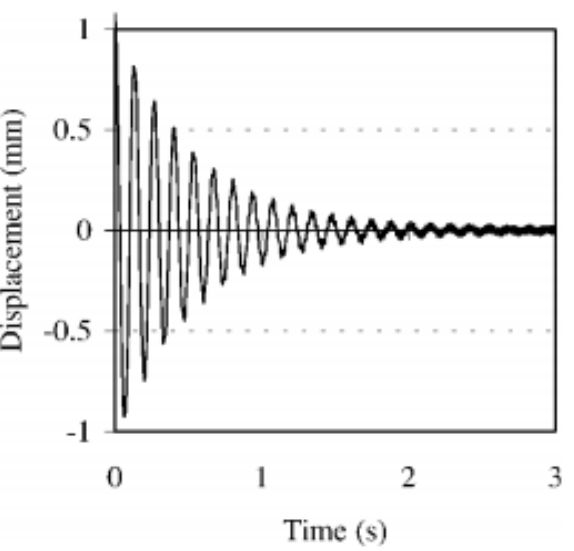

(b) Control voltage

Fig 3. Time history of the central displacement and control voltage.

The free vibration of the smart plate is caused by the sudden removal of a force of $3.5 \mathrm{~N}$ acted on the point $(0.4,0.3)$. Using the modal actuator and modal sensor and taking the control gain has $7 \times 10^{5}$, the modal control is performed, and the five controlled modes are shown in Fig. 3. The time history of the central displacement of the plate and control voltage applied on the modal actuator during control are presented in Figs. $4 \mathrm{a}$ and $4 \mathrm{~b}$, respectively. The results show that all of the target modes of the smart plate have been effectively controlled neurons.

\section{Summary}

A new practical method to design the modal actuator/sensor is given for modal control of smart plates by modulating the thickness distribution of the piezoelectric layer. If the stiffness and mass of the piezoelectric layer are considered, the thickness distribution of the modal actuator/sensor can be calculated by an iteration procedure based on a finite element model. In this way the present designing method for modal actuator and modal sensor can be applied to both one-dimensional and two-dimensional structures, such as beams, plates, and shells.

\section{References}

[1]. Zhu Xiaoping, Tao Baoji, et al. Vibration adaptive control and experimental research of piezoelectric motor truss structure. Vibration, test and diagnosis, 1998, 18 (2): 98 102.

[2]. Nia run rabbit, Shao Chengdu. Electromechanical coupling dynamic analysis and vibration control of intelligent trusses. Journal of vibration engineering, 1997, 10 (2): 119 124.

[3]. Li Jumbo, Liu Hua, Zhang Ling. Research on active component placement in dynamic testing of adaptive truss structures. Vibration, test and diagnosis, 1998, 18 (3): 184 189.

[4]. Sun Kang, Zhang Fugue. Piezoelectricity. Beijing: National Defence Industry Press, 1984.

[5]. GU Yuan Xian, Cheng Guangdong. Research and application of numerical methods for structural shape optimization design. Computational structural mechanics and its applications, 1993, 10 (3): 321 335.

[6]. GU Y X, Cheng G Structural modelling and sensitivity analysis of, sensitivity 1993, 6 (1): 29 37.

[7]. GU Y X, Kang Z et al. Dynamic sensitivity analysis and optimum, analysis 1998, 6 (1): 31 40.

[8]. Li Dengue. Dynamic analysis and fuzzy vibration of large flexible space truss structure, Dynamic control [M]. Beijing: Science Press, 2008.

[9]. Shao Chengdu, Zoo Zhengzhou. Electromechanical coupling dynamic analysis of Intelligent Trusses And vibration control. Journal of vibration engineering, 1997, 10 (2): 119 - 124. 